\title{
Toward a Deeper Understanding of Enzyme Reactions Using the Coupled ELF/NCl Analysis: Application to DNA Repair Enzymes
}

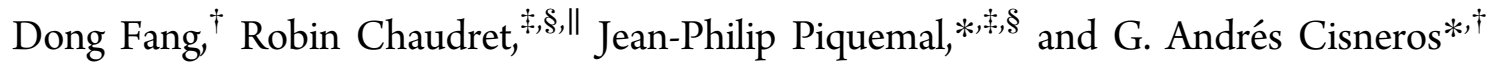 \\ ${ }^{\dagger}$ Department of Chemistry, Wayne State University, 5101 Cass Ave., Detroit, Michigan 48202, United States \\ ${ }^{\ddagger}$ UPMC Univ Paris 06, UMR 7616 Laboratoire de Chimie Théorique, Case Courrier 137, 4 Place Jussieu, F-75005, Paris, France \\ ${ }^{\S}$ CNRS, UMR 7616, Laboratoire de Chimie Théorique, Case Courrier 137, 4 Place Jussieu, F-75005, Paris, France
}

\section{Supporting Information}

ABSTRACT: The combined Electron Localization Funtion (ELF)/ Noncovalent Interaction (NCI) topological analysis (Gillet et al. J. Chem. Theory Comput. 2012, 8, 3993) has been extended to enzymatic reaction paths. We applied ELF/NCI to the reactions of DNA polymerase $\lambda$ and the $\varepsilon$ subunit of DNA polymerase III. ELF/NCI is shown to provide insights on the interactions during the evolution of enzymatic reactions including predicting the location of TS from structures located earlier along the reaction coordinate, differential metal coordination, and on barrier differences with two different cations.

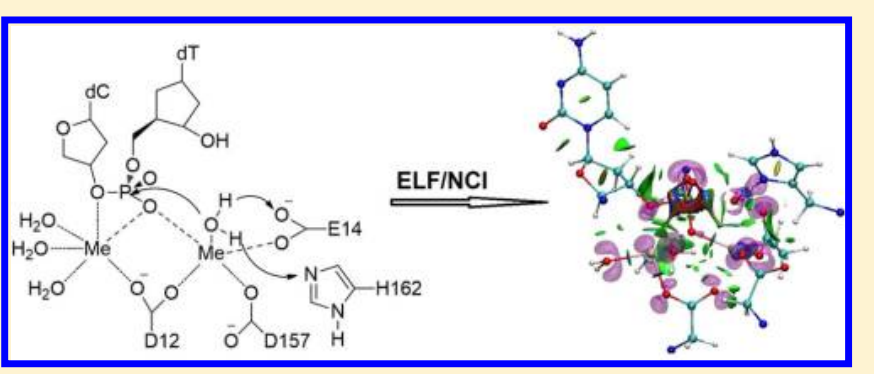

$\mathrm{D}$ NA polymerases and exonucleases play an important role in DNA replication and repair. ${ }^{1,2}$ DNA polymerases catalyze the addition of incoming deoxyribonucleotides (dNTP) to a nascent DNA chain. According to the primary structure of their catalytic subunits, DNA polymerases are categorized into several different families. ${ }^{3}$ Human DNA polymerase $\lambda$ is a member of the $\mathrm{X}$ family and has been implicated in the gap filling and end joining processes involved in the repair of DNA double-strand breaks and in base excision repair of damaged bases. ${ }^{4,5}$ Exonucleases catalyze the inverse reaction of polymerases, i.e., the excision of DNA bases. As a catalytic subunit of E. coli DNA polymerase III, subunit $\varepsilon$ accounts for the proofreading exonuclease activity by catalyzing the removal of newly incorporated mispaired nucleotides. ${ }^{6-8}$

Recently, some of us introduced a coupling of two quantum interpretative techniques using both the Electron Localization Function $(\mathrm{ELF})^{9-11}$ and the Noncovalent Interaction $(\mathrm{NCI})^{12,13}$ index to study reaction mechanisms through a partition of the electronic density. This combined ELF/NCI analysis was initially applied to investigate in detail prototypical organic reactions ${ }^{14}$ since this cross interpretative approach enables the following of the full range of interactions within a complex system. Indeed, ELF/NCI is able to simultaneously unravel regions of strong electron pairing (atoms and bonds) using ELF and regions of low density associated with low density reduced gradient, revealing weak interactions using NCI. This approach has been automated and gives chemically intuitive and visual results to describe chemical reactions involving multiple steps. ${ }^{14}$ In this contribution, we propose the first application of this technique to large systems of biological interest using hybrid QM/MM (Quantum Mechanics/Molecular Mechanics) methods. To this end, we have applied ELF/
NCI analysis to study the reactions catalyzed by DNA polymerase $\lambda(\operatorname{Pol} \lambda)$ and the $\varepsilon$ subunit of PolIII $(\varepsilon)$. The reaction paths associated with the catalytic mechanisms of these two enzymes have been studied in detail by some of us previously using QM/MM simulations. ${ }^{15,16}$ In both cases, two divalent metal cations are required for catalysis. Both DNA polymerases and exonucleases can employ different metals for catalysis. ${ }^{17-19}$ In our previous studies, we have investigated the role of $\mathrm{Mg}^{2+}$ and $\mathrm{Mn}^{2+}$ for the reaction mechanism catalyzed by both enzymes. ${ }^{15,16}$ In addition, we have recently used ELF to investigate metal ion mutagenicity in the synthesis of DNA by Pol $\lambda .^{17}$

The ELF function was originally proposed to measure the electron localization in atomic and molecular systems based on the Hartree-Fock formalism ${ }^{9}$ and subsequently extended to DFT. ${ }^{20}$ Similarly to the Bader and Austen's topological analysis of the electron density, ${ }^{21}$ the ELF values can also be treated as a continuous and differentiable scalar field in 3D space. The maxima of ELF (critical points) are named attractors and are located on atoms, bonds, and lone pairs following chemical intuition. Consequently, the molecular space can be divided into regions, named basins, that respectively contain all points whose ELF gradient field converges toward the same attractor. The different basins do not overlap, and the surface separating two basins is called a separatrix. Depending on the electrons they refer to, the different basins can be denoted as $\mathrm{C}()$ for core electrons or $\mathrm{V}()$ for valence electrons. Core basins are defined as belonging to a single nucleus. Valence basins may belong to a single atom and are termed monosynaptic (e.g., lone pairs) or

Received: February 19, 2013

Published: April 23, 2013 


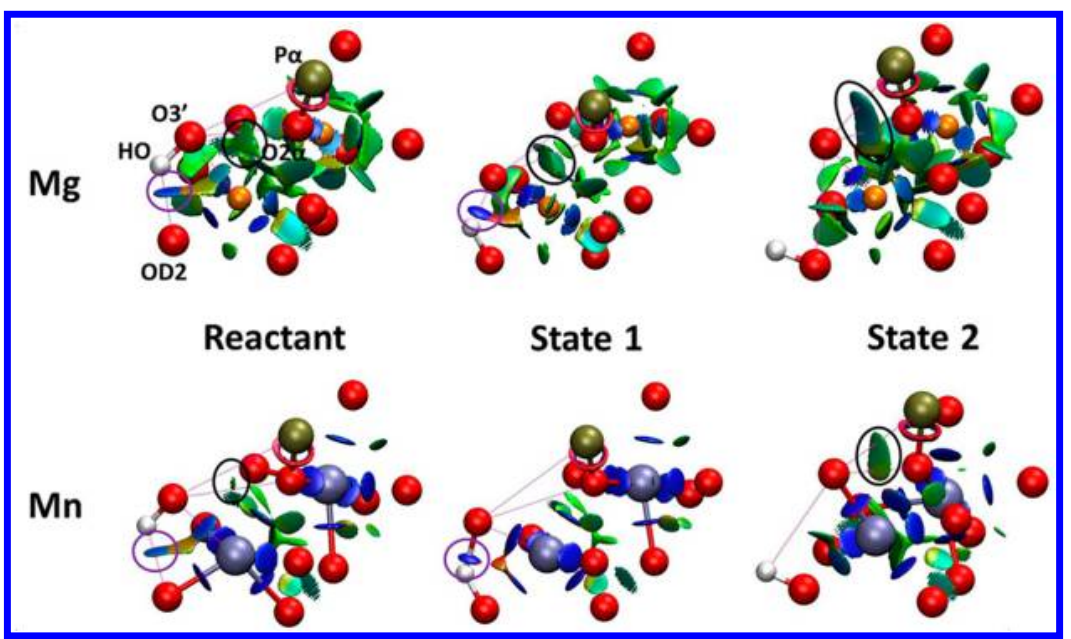

Figure 1. NCI analysis for the reactant, TS (state 1), and transphosphorilation structure (state 2) of Pol $\lambda$ with $\mathrm{Mg}^{2+}$ and $\mathrm{Mn}^{2+}($ the isovalue is 0.4 au, and the color scale is $-0.04 \mathrm{au}<\operatorname{sign}(\lambda 2) \rho<0.04 \mathrm{au})$.

may belong to two (or three) atoms and are termed disynaptic (e.g., bonds).

Recently, a third category has been proposed; subvalence electrons have been proposed to be the outer-shell "core" electrons of specific metal cations. ${ }^{18}$ Basins associated with chemical bonds between atoms $\mathrm{X}$ and $\mathrm{Y}$ are denoted as $\mathrm{V}(\mathrm{X}, \mathrm{Y})$. Furthermore, the ability to divide the molecular space into regions allows not only the computation of integrated properties, such as charge and volume, but also local electrostatic multipoles or Fukui functions over these regions. $^{18,22}$

The $\mathrm{NCI}^{12,13}$ index enables the study of the domains of the electronic density associated with weak interactions that exhibit both low electron density and low reduced density gradient(s):

$$
s(\rho)=\frac{|\nabla \rho|}{2\left(3 \pi^{2}\right)^{1 / 3} \rho^{4 / 3}}
$$

where $\rho$ is the electron density. By multiplying the density by the sign of the second eigenvalue of the density Hessian $\left(\lambda_{2}\right)$, one can distinguish the strength and the attractive or repulsive nature of the interactions. ${ }^{23}$ In the noncovalent region, an arbitrary color code was chosen so that, for NCI surfaces: The red color is used for regions with a positive $\lambda_{2}$ that are associated with steric repulsion. The blue color is used for domains with a negative $\lambda_{2}$, therefore indicating relatively strong attraction (for example, H-bond regime). The green color is used for regions of very weak reduced density gradient, associated with van der Waals interactions. The ELF calculations were carried out using the TopMod software. ${ }^{24}$ The $\mathrm{NCI}^{12,13}$ results were calculated with the NCIPLOT program. ${ }^{12,13}$ Details about the computations are available in the Supporting Information.

The ELF analysis of the critical structures for the reaction catalyzed by Pol $\lambda$ have been presented in a previous study. ${ }^{17}$ Herein, only their NCI surfaces are discussed (the combined ELF/NCI surfaces are shown in Figure S1). The NCI analysis shows the evolution of the different interactions along the reaction path (see Figure 1). Interestingly, the interactions related to the two legs of the path are already visible at the reactant state. As expected, the strong interaction between $\mathrm{HO}$ and OD2 from Asp490 (circled in purple) clearly appears at the reactant state and evolves along the first part of the reaction (see reactant structure in Scheme 1 for atom labels). After the
Scheme 1. Reaction Mechanism for the Reaction Catalyzed by $\operatorname{Pol} \lambda^{a}$

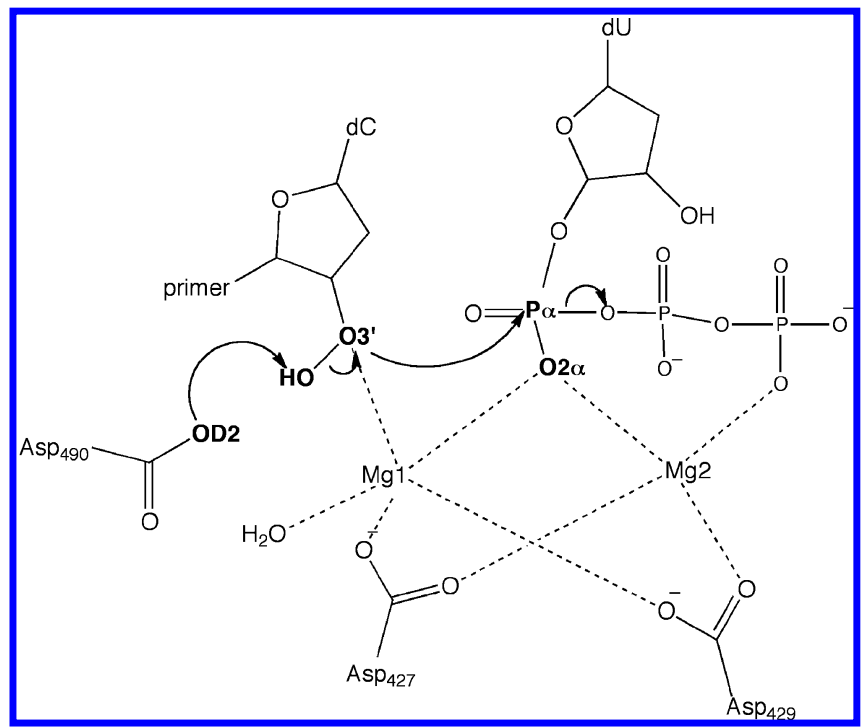

${ }^{a}$ The atom labels used in the text are denoted in the reactant structure.

proton transfer, a strong hydrogen bond between $\mathrm{O}^{\prime}$ and $\mathrm{HO}$ remains clearly visible. Concerning the second part of the reaction (transphosphorylation), the interaction between $\mathrm{O}^{\prime}$ and $\mathrm{O} 2 \alpha$ (circled in black), that can be seen from the reactant state, will evolve along the reaction path to give rise to the $\mathrm{P} \alpha-$ O3' bond at the product state. The strength of this basin is characteristic of the van der Waals interactions at the reactant state; it will however evolve into a delocalized double basin whose attractive part is characteristic of the $\mathrm{P} \alpha-\mathrm{O}^{\prime}$ attraction and repulsive part of the $\mathrm{O}^{\prime}-\mathrm{O} 2 \alpha$ electrostatic repulsion. This is similar to the evolution of the $\mathrm{C}-\mathrm{C}$ interaction into a $\mathrm{C}-\mathrm{C}$ bond and the development of ring tension during the electrocyclization of butadiene as previously described. ${ }^{14}$

The comparison between the $\mathrm{Mg}^{2+}$ and $\mathrm{Mn}^{2+} \mathrm{NCI}$ topologies shows some important differences. The $\mathrm{O}^{\prime}-\mathrm{Mg}$ interaction is weaker than the $\mathrm{O}^{\prime}-\mathrm{Mn}$ one. Conversely, the $\mathrm{O}^{\prime}-\mathrm{O} 2 \alpha$ interaction is weaker (and so is $\mathrm{O}_{3}{ }^{\prime}-\mathrm{P} \alpha$ ) in the $\mathrm{Mn}^{2+}$ structure than in the $\mathrm{Mg}^{2+}$ case. Indeed, since $\mathrm{Mg}^{2+}$ is a hard cation and $\mathrm{Mn}^{2+}$ is a soft one, the $\mathrm{O}^{\prime}-\mathrm{Mg}$ interaction 
involves less electrons than the $\mathrm{O}^{\prime}-\mathrm{Mn}$. Therefore, more electrons are accessible to the $\mathrm{O}-\mathrm{H}$ bond and the other lone pair in the $\mathrm{Mg}$ case, suggesting that (i) the OD2-HO interaction is weaker because $\mathrm{HO}$ is less acidic and (ii) the $\mathrm{O}^{\prime}-\mathrm{O} 2 \alpha$ and $\mathrm{P} \alpha$ interaction is stronger. Finally, this is also consistent with our previous $\mathrm{QM} / \mathrm{MM}$ energetic results that showed that the barrier for proton transfer is higher when $\mathrm{Mg}^{2+}$ is present in the active site compared to $\mathrm{Mn}^{2+} .{ }^{17}$

As described above, $\varepsilon$ catalyzes the excision of a nucleotide from DNA (see Scheme 2). Figure 2 shows the ELF and NCI

\section{Scheme 2. Reaction Mechanism for the $\varepsilon$ Subunit $^{a}$}

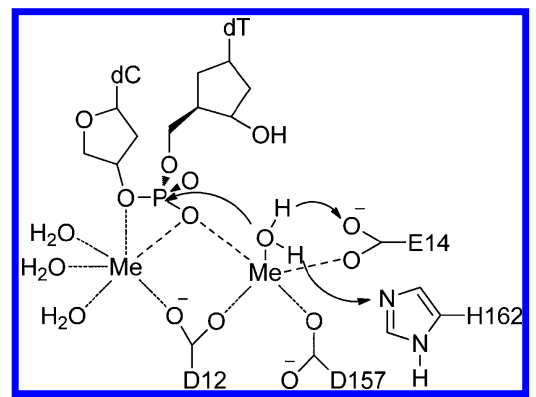

${ }^{a}$ In the first step, a proton is transferred from the ordered water to H162 to activate the nucleophile. Subsequently, the oxygen performs a nucleophilic attack to break the phosphoesther bond coupled with a second proton transfer to E14. ${ }^{16}$

surfaces of the critical points on the path for the reaction catalyzed by the $\varepsilon$ subunit with $\mathrm{Mg}^{2+}$ and $\mathrm{Mn}^{2+}$. As shown in Figure S2, the basins of the $\mathrm{Mg}^{2+}$ ions are spherical as expected. Conversely, there are several basins around each of the $\mathrm{Mn}^{2+}$. This has been observed previously in the context of other metalloproteins and has been termed "subvalence splitting."17,18 It has been shown that the splitting of the subvalence in metal cations is due to the partial covalency between the metal cation and the ligands around it $^{18}$ and may be related to some biological features of the metal cations. ${ }^{17,18}$

Tables S1 and S2 (see Supporting Information) confirm the splitting of the electronic density of the two $\mathrm{Mn}^{2+} \mathrm{s}$ into several distinct basins, while that of the $\mathrm{Mg}^{2+}$ cations shows only one basin. This suggests that the interactions between the $\mathrm{Mn}^{2+}$ and the ligands in their first coordination shell are stronger than those of the $\mathrm{Mg}^{2+}$ and its corresponding ligands. For the $\mathrm{Mn}^{2+}$ cation, the catalytic metal ( $\mathrm{Me} 2$, see Figure 3) has five

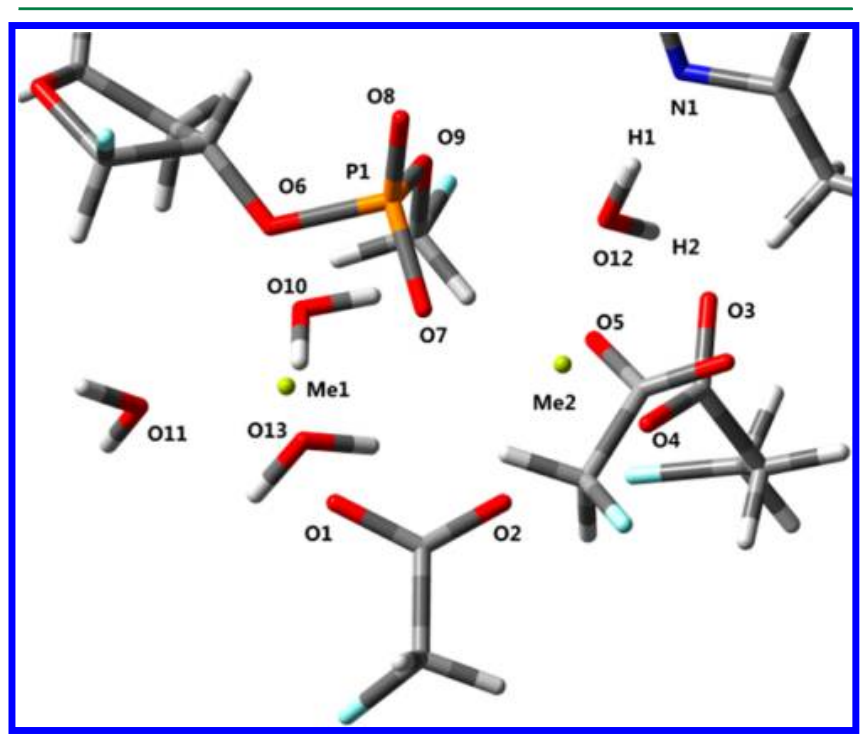

Figure 3. Numbering of the atoms in $\varepsilon$ that are used in the ELF calculations.

subvalence basins while the binding metal (Me1) has six subvalence basins. In this special case, the number of the

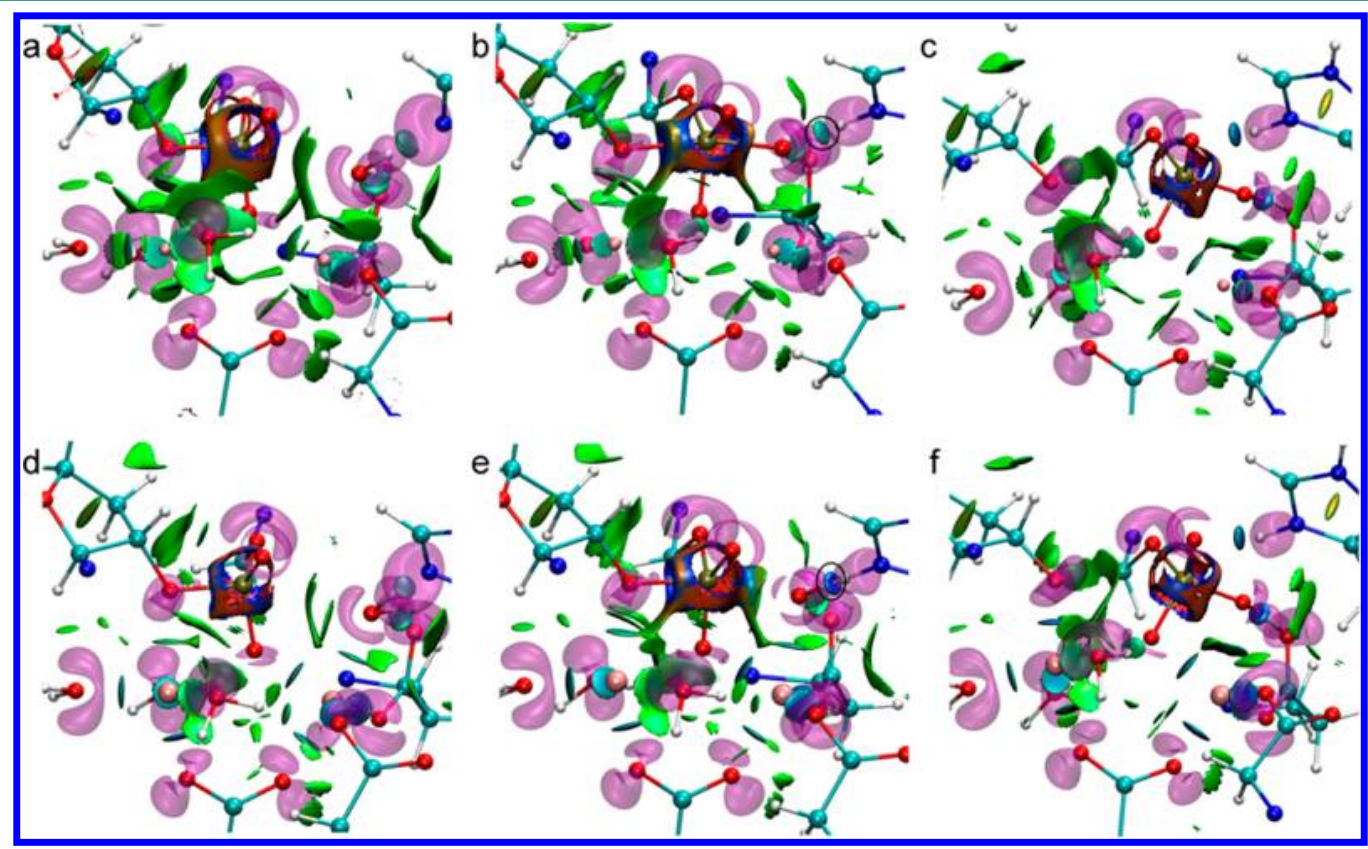

Figure 2. Combined ELF and NCI surfaces of the critical structures for the reaction catalyzed by the $\varepsilon$ subunit with $\mathrm{Mg}^{2+}$ and $\mathrm{Mn}^{2+}$. Only atoms subjected to the ELF analysis are shown; the rest of the QM subsystem is omitted for clarity. Panels $a$, b, and $\mathrm{c}$ show the reactant, TS, and product for $\mathrm{Mg}^{2+}$, respectively. Panels d, e, and f correspond to the reactant, TS, and product for $\mathrm{Mn}^{2+}$, respectively. The isovalue for ELF is 0.87 , and for $\mathrm{NCI}$ it is $0.5 \mathrm{au}$ with the color scale -0.1 au $<\operatorname{sign}(\lambda 2) \rho<0.1$ au. 


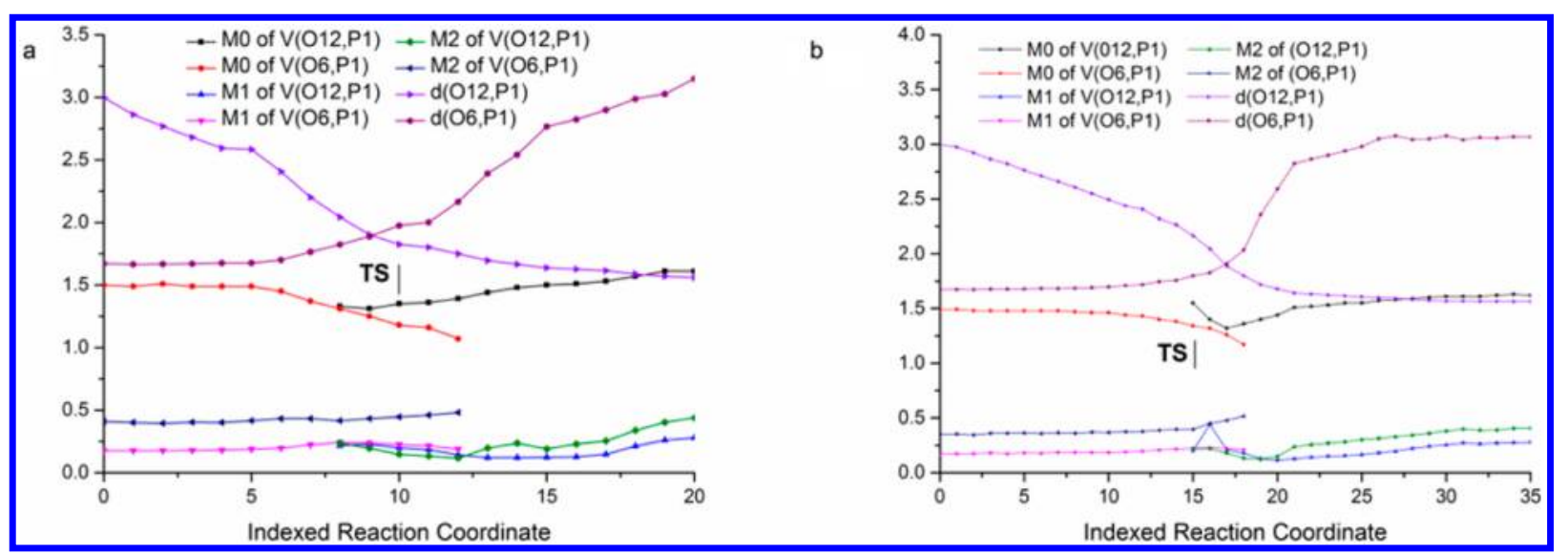

Figure 4. Distributed moments, $\mathrm{M}_{0}$ (population), $\mathrm{M}_{1}$ (first moment), and $\mathrm{M}_{2}$ (second moment) of $\mathrm{V}(\mathrm{O} 6, \mathrm{P} 1)$ and $\mathrm{V}(\mathrm{O} 12, \mathrm{P} 1)$ and the distances $\left(d(\mathrm{O} 6, \mathrm{P} 1)\right.$ and $d(\mathrm{O} 12, \mathrm{P} 1)$, in $\AA$, along the reaction ( $\mathrm{a}, \mathrm{b}$ are for the reactions catalyzed by $\mathrm{Mg}^{2+}$ and $\mathrm{Mn}^{2+}$, respectively).

splitting subvalence basins for each metal ion is equal to the number of ligands in the first coordination shell for $\mathrm{Mn}^{2+}$. In addition, the number of basins in the subvalence for the metals remains constant during the progress of the reaction.

A multipolar expansion of the electron density of each ELF basin can provide further insight into the changes in the basins along the reaction thanks to the evaluation of local electrostatic moments. In this case, the first and second moments of each basin for both metal cations show only slight changes. However, ELF can also provide insights on the bonds that break (between $\mathrm{O} 6$ and P1) and form (between $\mathrm{O} 12$ and P1) during the reaction by analyzing the evolution of the basins associated with these bonds. Figure 4 shows the distance evolution $\mathrm{O} 6-\mathrm{P} 1$ and $\mathrm{O} 12-\mathrm{P} 1$ and the multipolar decomposition evolution (population, first and second moments, $\mathrm{M}_{0}$, $\mathrm{M}_{1}$, and $\mathrm{M}_{2}$, respectively) of two bond basins, $\mathrm{V}(\mathrm{O} 6, \mathrm{P} 1)$ and $\mathrm{V}(\mathrm{O} 12, \mathrm{P} 1)$ for the $\mathrm{Mn}^{2+}$ and $\mathrm{Mg}^{2+}$ catalyzed reactions. In the case of the $\mathrm{Mg}^{2+}$ catalyzed reactions, the TS is late in the reaction coordinate, with a longer distance for the breaking bond, $d(\mathrm{O} 6-\mathrm{P} 1)$, than for the forming bond, $d(\mathrm{O} 12-\mathrm{P} 1)$. When $\mathrm{Mn}^{2+}$ is in the active site, the TS is located earlier in the path with a significantly shorter distance for the breaking bond and a longer distance for the forming bond. In particular, the two distances and the populations of these two basins change dramatically around the TS.

The most significant change is in the population of the basins for the forming and breaking bonds. Interestingly, the appearance of the $\mathrm{V}(\mathrm{O} 12, \mathrm{P} 1)$ basin (corresponding to the forming bond) shows significant differences between the two cations. In the case of $\mathrm{Mg}^{2+}$, the basin appears before the system reaches the TS with a small population of 1.25 , which increases continuously as the reaction reaches the TS and plateaus as the reaction continues to the product. For the $\mathrm{Mn}^{2+}$ case, the basin only arises at the TS with a much larger population of around 1.7, which shows a marked decrease and then an increase as the reaction proceeds. This population change indicates that the $\mathrm{Mn}^{2+}$ cation induces a larger charge transfer on the three atoms involved in these two basins. The earlier TS and larger population change in the $\mathrm{Mn}^{2+}$ catalyzed reaction help explain the lower energy barrier compared to the $\mathrm{Mg}^{2+}$ catalyzed reaction. The first and second moments $\left(\mathrm{M}_{1}\right.$ and $\mathrm{M}_{2}$, respectively) for both basins show only modest variations along the path, indicating only a small change in polarization on these basins.
Since most of the time the interactions between the metal and its ligands are not traditional covalent bonding, no typical ELF basins for covalent bonds can be found. In this case, NCI plays an important role in the deep understanding of this type of intermolecular interaction. Indeed, the number of NCI surfaces is able to indicate the coordination number of $\mathrm{Mg}^{2+}$ and $\mathrm{Mn}^{2+}$ as well. For $\varepsilon$, the catalytic metal (Me2) is surrounded by five NCI surfaces for both cations, which suggests a penta-coordination in both cases. This is consistent with our previous results for the reaction path calculation, which showed that this penta-coordination on the catalytic metal polarizes the water to facilitate the first deprotonation. ${ }^{16}$ In contrast, the nucleotide binding metal (Me1) is surrounded by six NCI surfaces for both $\mathrm{Mg}^{2+}$ and $\mathrm{Mn}^{2+}$.

For the same metal center, different ligands produce NCI surfaces with different color depths, which can differentiate the strength of the interactions. Generally speaking, the NCI surfaces between ligands and the metal centers for $\mathrm{Mn}^{2+}$ are bluer than the ones in $\mathrm{Mg}^{2+}$. This means that the interactions for $\mathrm{Mn}^{2+}$ are stronger than $\mathrm{Mg}^{2+}$, which is in accord with the lower energy barrier for $\mathrm{Mn}^{2+}$. For the metal in the reactant structure, the surfaces for $\mathrm{O} 2, \mathrm{O} 7, \mathrm{O} 5$, and $\mathrm{O} 4$ are bluer than the one for the oxygen of the nucleophilic water (O12). For Me1, O6 has the weakest interaction compared to the other ligands coordinated to Mel. Compared to the reactant, the surface between P1 and $\mathrm{O} 12$ in the TS becomes bluer, indicating the increasing attraction between these atoms as the bond forms. In addition, the blue surfaces between the transferred hydrogen atoms $(\mathrm{H} 1, \mathrm{H} 2)$ show strong hydrogen bonding (circled in black, Figures $2 \mathrm{~b} / \mathrm{e}$ ) with the corresponding oxygen atoms (O8, O12).

Thus, these two analyses are consistent with each other. Furthermore, regarding the proton $\mathrm{H} 1$, which is transferred from $\mathrm{O} 12$ to N1, in both TS structures it shows a shared basin with the lone electron pair of N1, which means the proton transfer from the nucleophilic water to N1 has already started. The distance between $\mathrm{H} 1$ and $\mathrm{N} 1$ is $1.06 \AA$ for the $\mathrm{Mg}^{2+}$ catalyzed structure. For $\mathrm{Mn}^{2+}$, the distance is $1.14 \AA$, close to the value for $\mathrm{Mg}^{2+}$. However, for $\mathrm{Mg}^{2+}$, the population of this shared basin is about 2.72. In comparison, for $\mathrm{Mn}^{2+}, \mathrm{H} 1$ forms a "bond-like" basin with $\mathrm{N}$ with a population of 0.86 . The rest of the lone pair of $\mathrm{N} 1$ forms an extra basin with a population that is 1.07. The NCI surfaces between $\mathrm{H} 1$ and $\mathrm{O} 12$ (circled in black in Figure 2) are much bluer for $\mathrm{Mn}^{2+}$ than for $\mathrm{Mg}^{2+}$, indicating a stronger interaction between these atoms for $\mathrm{Mn}^{2+}$ 
than for $\mathrm{Mg}^{2+}$. This is in good agreement with the smaller basin population for the $\mathrm{Mn}^{2+}$ structure.

In general, in terms of NCI surfaces, $\mathrm{Mn}^{2+}$ cations have bluer NCI surfaces between the ligands than their corresponding $\mathrm{Mg}^{2+}$ cations. With regards to ELF, $\mathrm{Mn}^{2+}$ possesses more splitting basins. Both of them are suggestive of the stronger ligand interactions for $\mathrm{Mn}^{2+}$, which is in agreement with its lower reaction barrier than $\mathrm{Mg}^{2+} \cdot{ }^{15,16}$

As shown in the movie in the Supporting Information, the NCI surfaces are able to help predict the forthcoming forming or breaking of bonds, and the ELF basins provide the details of existing bonds. When the bonds (for example, the bonds between $\mathrm{O} 12$ and $\mathrm{P} 1, \mathrm{H} 1$ and $\mathrm{N} 1$, and $\mathrm{H} 2$ and $\mathrm{O} 3$ ) begin to form as the reaction progresses, NCI surfaces appear and become bluer; then they turn into rings (due to the cutoff for the electron density, see Supporting Information) and enlarge gradually, concurrent with the appearance of ELF basins. When the bonds begin to break (for instance, O6-P1 and $\mathrm{H} 1-\mathrm{O} 12$ ), the ELF basins become smaller, coupled to the emergence of NCI surfaces. Similarly to the findings of Gillet et al., ${ }^{14}$ the ELF attractors and the NCI interaction critical points (the holes) overlap. That is, ELF basins appear where NCI surfaces disappear, and vice versa providing ways to anticipate transition states.

In summary, we have demonstrated the applicability of the recently developed ELF/NCI topological approach to complex enzyme reactions involving large biological systems treated at the QM/MM level. Once again, ELF and NCI have been found to be highly complementary, giving information on bond formation and breaking, and on weak interactions thanks to $\mathrm{NCI}$, and on the reorganization of existing bonds and lone pairs with ELF. In addition, NCI was shown to be able to provide further insights into the factors governing the lowering of the reaction barrier in $\mathrm{Pol} \lambda$ when $\mathrm{Mn}^{2+}$ is in the active site compared to $\mathrm{Mg}^{2+}$. In the case of the $\varepsilon$ subunit, both ELF and NCI proved to be useful in understanding the reasons for the lower barrier in the $\mathrm{Mn}^{2+}$ catalyzed reaction compared to $\mathrm{Mg}^{2+}$. Moreover, the NCI analysis provided further support for the unusual penta-coordination observed in $\varepsilon$ for the cation in the metal 2 position, which enables the activation of the nucleophile. Thus, the combination of these two techniques can be a useful and powerful tool to study the interaction changes along the enzyme reactions involved with metals. Finally, extending on the findings of Gillet et al., ${ }^{14}$ the ELF attractors and the NCI interaction critical points overlap as NCI critical points appear to be precursors of ELF attractors, providing new strategies to understand and predict the location of transition states in enzymes.

\section{ASSOCIATED CONTENT}

\section{S Supporting Information}

Expanded computational details, combined ELF/NCI surfaces for Pol $\lambda$ structures, ELF basins of the metals, and animations of the evolution of ELF and NCI surfaces along the reaction path for reactions catalyzed by the $\varepsilon$ subunit. This information is available free of charge via the Internet at http://pubs.acs.org/.

\section{AUTHOR INFORMATION}

\section{Corresponding Author}

*E-mail: jpp@lct.jussieu.fr (J.P.P.), andres@chem.wayne.edu (G.A.C.).

\section{Present Address}

"IFP Energies Nouvelles, 1\&4, Avenue des Bios-Préau, 92852 Rueil-Malmaison Cedex, France

Notes

The authors declare no competing financial interest.

\section{ACKNOWLEDGMENTS}

Financial support from Wayne State University to G.A.C., and from the French National Research Agency (Grant ANR-08BLAN-0158) to J.P.P., as well as computing time from Wayne State C\&IT are gratefully acknowledged.

\section{REFERENCES}

(1) Loeb, L. A.; Monnat, R. J. Nat. Rev. Genet. 2008, 9, 594-604.

(2) Thomas, K. R.; Olivera, B. M. J. Biol. Chem. 1978, 253, 424-429.

(3) Bebenek, K.; Kunkel, T. A. Adv. Protein Chem. 2004, 69, 137165

(4) Garcia-Diaz, M.; Bebenek, K.; Gao, G.; Pedersen, L. C.; London, R. E.; Kunkel, T. A. DNA Repair 2005, 4, 1358-1367.

(5) Garcia-Diaz, M.; Bebenek, K.; Krahn, J. M.; Pedersen, L. C.; Kunkel, T. A. Cell 2006, 124, 331-342.

(6) Brenowitz, S.; Kwack, S.; Goodman, M. F.; O’Donnell, M.; Echols, H. J. Biol. Chem. 1991, 266, 7888-7892.

(7) McHenry, C. S. J. Biol. Chem. 1991, 266, 19127-19130.

(8) Derbyshire, V.; Pinsonneault, J. K.; Joyce, C. M. Methods Enzymol. 1995, 262, 363-385.

(9) Becke, A. D.; Edgecombe, K. E. J. Chem. Phys. 1990, 92, 53975403.

(10) Piquemal, J. P.; Pilmé, J.; Parisel, O.; Gérard, H.; Fourré, I.; Bergès, J.; Gourlaouen, C.; De La Lande, A.; Van Severen, M. C.; Silvi, B. Int. J. Quantum Chem. 2008, 108, 1951-1969.

(11) Silvi, B.; Savin, A. Nature 1994, 371, 683-686.

(12) Contreras-García, J.; Johnson, E. R.; Keinan, S.; Chaudret, R.; Piquemal, J.-P.; Beratan, D. N.; Yang, W. J. Chem. Theory Comput. 2011, 7, 625-632.

(13) Johnson, E. R.; Keinan, S.; Mori-Sánchez, P.; Contreras-García, J.; Cohen, A. J.; Yang, W. J. Am. Chem. Soc. 2010, 132, 6498-6506.

(14) Gillet, N.; Chaudret, R.; Contreras-García, J.; Yang, W.; Silvi, B.; Piquemal, J.-P. J. Chem. Theory Comput. 2012, 8, 3993-3997.

(15) Cisneros, G. A.; Perera, L.; García-Díaz, M.; Bebenek, K.; Kunkel, T. A.; Pedersen, L. G. DNA Repair 2008, 7, 1824-1834.

(16) Cisneros, G. A.; Perera, L.; Schaaper, R. M.; Pedersen, L. C.; London, R. E.; Pedersen, L. G.; Darden, T. A. J. Am. Chem. Soc. 2009, $131,1550-1556$.

(17) Chaudret, R.; Piquemal, J.-P.; Cisneros, G. A. Phys. Chem. Chem. Phys. 2011, 13, 11239-11247.

(18) de Courcy, B.; Pedersen, L. G.; Parisel, O.; Gresh, N.; Silvi, B.; Pilmé, J.; Piquemal, J. P. J. Chem. Theory Comput. 2010, 6, 1048-1063.

(19) Sirover, M. A.; Loeb, L. A. Science 1976, 194, 1434-1436.

(20) Savin, A.; Jepsen, O.; Flad, J.; Andersen, O.; Preuss, H.; von Schnering, H. Angew. Chem., Int. Ed. 1992, 31, 187-188.

(21) Bader, R. F. W.; Austen, M. A. J. Chem. Phys. 1997, 107, 42714285.

(22) Pilmé, J.; Piquemal, J.-P. J. Comput. Chem. 2008, 29, 14401449.

(23) Contreras-Garcia, J.; Yang, W.; Johnson, E. R. J. Phys. Chem. A 2011, 115, 12983-12990.

(24) Noury, S.; Krokidis, X.; Fuster, F.; Silvi, B. Comput. Chem. 1999, 23, 597-604. 